Les Cahiers du Gres

\section{Le genre et l'ethnicité : les critères voilés de la délégation des actes en santé}

\section{Marguerite Cognet et Laurent Raigneau}

Volume 3, numéro 1, printemps 2002

URI : https://id.erudit.org/iderudit/009428ar

DOI : https://doi.org/10.7202/009428ar

Aller au sommaire du numéro

Éditeur(s)

Groupe de recherche ethnicité et société

CEETUM

ISSN

1499-0431 (imprimé)

1499-044X (numérique)

Découvrir la revue

Citer cet article

Cognet, M. \& Raigneau, L. (2002). Le genre et l'ethnicité : les critères voilés de la délégation des actes en santé. Les Cahiers du Gres, 3(1), 25-38.

https://doi.org/10.7202/009428ar

citer cet article
Les Cahiers du Gres Série Diversité urbaine Groupe de Recherche Ethnicte et Sodet 
Marguerite COGNET, Ph.D.

Chercheure FQRSC

CRF - CLSC Côte-des-Neiges, Centre affilié universitaire McGill

URMIS, Paris VIII

Professeure associée

Département de Communication

Université du Québec à Montréal

Laurent RAIGNEAU, M.Sc.

Professionnel de recherche

CRF - CLSC Côte-des-Neiges, Centre

affilié universitaire McGill

\section{Le genre et l'ethnicité : les critères voilés de la délégation des actes en santé}

L'organisation du travail de la santé se caractérise par l'importance des zones où les uns et les autres partagent des compétences. Autour du patient et de la maladie, du diagnostic à la guérison en passant par les soins, se dessinent une série d'actes et se succèdent divers acteurs professionnels. L'histoire des professions soignantes s'est faite et continue de se faire au sein de ce système qui paraît d'autant mieux orchestré qu'il est structuré par une forte hiérarchisation. Dans un système de santé les zones de partage des compétences, instrumentalisées comme espaces d'incertitude, deviennent aussi des zones de pouvoir et d'enjeu (Crozier et Friedberg, 1977) où se négocient les limites des compétences de l'Autre, la préservation de son propre espace et l'extension de celui-ci (Cognet, 1999). En même temps que les uns concourent à s'approprier tel ou tel acte pour en réclamer des compétences effectives et donc une reconnaissance légitime, les autres le revendiquent comme marqueur de territoires réservés ou sur lesquels ils veulent conserver des prérogatives et négocier sous certaines conditions (Desrosiers, 2001 ; Dussault et Potvin, 1983 ; Cohen, 1995 ; Trabacchi, 2000). Parallèlement à cette «circulation» des actes, on assiste à la définition subséquente de leur valeur par les uns et les autres, valeur qui participe de plain-pied à définir l'autorité même de ceux qui en sont les détenteurs. Dans ces luttes ou, plus exactement, cette compétition aux compétences, la légitimité à s'approprier celles-ci ou à l'inverse à déléguer celles-là, s'appuie surtout sur un système de références qui met en scène divers types d'appartenance : professionnelle ou disciplinaire, organisationnelle mais aussi de genre ou/et d'ethnicité. Ces appartenances mises en jeu le sont parfois explicitement parfois implicitement mais toujours de façon croisée.

Nous avons pour hypothèse de nos recherches que c'est dans cet étroit maillage des appartenances que se négocient les conflits et que naissent les consensus même si ceux-ci sont provisoires. Ces études ${ }^{1}$ ont pour objet les pratiques et les identités au travail de différentes catégories de professionnels de la santé (médecins, infirmiers, aides soignants, auxiliaires familiaux, etc.) ainsi que celles de différents segments existant en leur sein. Elles sont appréhendées de façon à pouvoir interroger les effets des formations, des positions occupées dans le système institutionnel de la santé ainsi que ceux plus diffus des appartenances liées aux genres et à l'ethnicité. Autrement dit, les pratiques sont analysées comme des ressources que les catégories et segments professionnels utilisent pour définir leurs territoires, délimiter leurs espaces de compétence, se situer chacun en regard des autres. Dans ce sens les pratiques professionnelles sont constitutives des identités au travail et l'appropriation des compétences qui leur sont inhérentes est au cœur des enjeux.

Le lecteur l'a sans doute pressenti, notre cadre d'analyse s'inscrit dans la lignée des travaux, désormais clas- 
siques, des interactionnistes de l'école de Chicago en commençant par Hughes (1958) pour qui toute analyse sociologique du travail en santé repose d'abord sur l'analyse de la division du travail. L'activité professionnelle quelle qu'elle soit, pour le travail en santé comme dans toute autre sphère, ne prend tout son sens que réintégrée dans l'ensemble dans lequel elle s'insère. II faut considérer en premier lieu le processus d'organisation dudit ensemble, à commencer par les procédures de distribution des tâches entre les individus et les groupes (Dubar, 1996). Cette approche a deux implications majeures pour qui s'intéresse aux professions et métiers ${ }^{2}$ de la santé. À un premier niveau, il faut considérer les activités concernant la santé dans l'ensemble des activités du corps social et c'est d'ailleurs le point de départ des sociologues de Chicago ; ensuite, c'est l'organisation du travail de la santé et sa division qui est à analyser. Sur ce point les études de Freidson (1984) sont fondatrices. Aucun groupe professionnel ne peut s'étudier séparément du système professionnel dont il est issu, pas plus que du contexte social et politique dans lequel il prend naissance, évolue et se maintient (Dubar et Tripier, 1998). L'organisation du travail en santé y compris la distribution des activités entre les groupes professionnels, résulte d'une alliance historique entre les intérêts d'individus occupant des positions de pouvoir (i.e. le corps médical) et ceux de la société en général représentée par l'État dont l'enjeu est la régulation sociale (Dubar et Tripier, 1998 ; Freidson, 1984). Notre propos s'articule autour de concepts clés : la division sociale du travail de la santé, l'ordre négocié de l'organisation hospitalière et des professions de la santé, la construction sociale de la maladie et des sujets malades.

En analysant les pratiques de la délégation des actes de soins au Québec, les règlements et les faits observables au travers de la « trajectoire » de quelques actes comme l'administration de médicaments, la prise des constantes (pouls, pression artérielle, etc.), l'insulinothérapie ou encore les curages et les touchers rectaux, parmi les catégories infirmières ${ }^{3}$, infirmières auxiliaires et auxiliaires familiales, nous verrons que les négociations autour des actes et des compétences - au-delà des actes eux-mêmes, de la technicité, de la dextérité ou encore des savoirs objectifs qu'ils requièrent - mettent en jeu des appartenances à des groupes tels que les institutions, les organisations professionnelles ou les services mais aussi des appartenances de genre et d'origines ethniques et sociales. Nous verrons aussi que la délégation de ces actes est à analyser dans le contexte des transformations des systèmes de santé consécutives aux politiques de réduction des coûts et dont les effets et les options ne sont pas sans relation avec une hiérarchisation des problèmes de santé et des populations soignées (Montgomery et al. 2001).

\section{Le genre et l'ethnicité dans les or- ganisations de la santé}

Diverses études ont montré que d'une division du travail médical à une division sexuée de ce même travail, il n'y a qu'un pas (Herzlich et al., 1993 ; Paicheler, 2000 ; Saillant, 1994). La part de femmes dans les différents grades professionnels est inversement proportionnelle au degré de prestige, d'autonomie ou, disons plus simplement, à l'ordre hiérarchique statutaire avec tout ce que cela implique, tant en termes de reconnaissance des savoirs propres que des conditions de travail et de rémunération. Même quand les femmes accèdent aux professions médicales, c'est dans les domaines les moins spécialisés (tels la médecine générale ou la médecine familiale) et où le prestige est loin de celui des spécialités nécessitant 
des technologies ultra-sophistiquées. Mais plus encore, ces mêmes recherches ont montré le lien entre les carrières professionnelles et les trajectoires sociales croisant non seulement le genre, l'origine sociale (Herzlich et al., 1993 ; Aïach et Fassin, 1994), l'origine géographique, mais aussi les appartenances ethniques et religieuses (Herzlich et al., 1993 ; Hughes, 1956 ; Solomon, 1961 ; Freidson, 1984). Nos propres recherches conduisent à cette même évidence : la répartition des emplois dans les organisations de santé suit une répartition non aléatoire à la fois par le genre et par le lieu de naissance des individus.

Au Québec, l'accès aux emplois dans les organisations de santé se distribue différemment entre les hommes et les femmes comme entre les nationaux et les étrangers. La part des hommes croît avec la hiérarchie et, si les femmes sont bien représentées chez les médecins (40\% au Québec) ${ }^{4}$, elles le sont moins dans les spécialités que dans la médecine familiale. En revanche, dans les emplois para-médicaux, les femmes sont largement sur-représentées : elles comptent pour $91 \%$ des effectifs infirmiers 5 , $83 \%$ de ceux des travailleurs sociaux 6 , $80 \%$ de ceux des auxiliaires familiales et sociales.

Outre la forte féminisation de la catégorie des auxiliaires familiales, notre enquête à Montréal ${ }^{7}$ met en évidence l'importance de la population immigrée $(45 \%)^{8}$. À partir de notre enquête par questionnaire, nous avons effectué plusieurs croisements de variables dont celles concernant le lieu de naissance des individus, ceux de leurs parents, les nationalités et la citoyenneté, les parcours migratoires, le genre et le type d'employeur. Avec un échantillon $(\mathrm{N}=181)^{9}$ d'individus en emploi dans trois CLSC et autant d'agences, ces données nous permettent de dresser un profil de la population des auxiliaires familiales actuellement en emploi dans ces entreprises montréalaises ${ }^{10}$. Apparaît une relation significative entre ces variables. Tout d'abord, nous observons que la répartition hommes/femmes dans cette catégorie d'emploi n'est pas exactement la même selon que les individus sont immigrés ou Canadiens ${ }^{11}$, qu'ils proviennent, eux (ou leurs parents), d'un pays à majorité noire ou blanche. Ainsi, alors que les hommes canadiens, nés de parents canadiens, ne représentent que $19 \%$ de la population canadienne de notre échantillon, les hommes immigrés comptent pour $21 \%$ de la population immigrée de l'échantillon. Les hommes originaires, eux-mêmes ou leurs parents, de pays à majorité noire occupent $23 \%$ des postes détenus par ce sous-groupe alors que les hommes blancs n'en représentent que $19 \%$ des cas. Aux vues de ces résultats, il semble que les hommes immigrés, en particulier les hommes immigrés noirs, subissent une plus forte attraction pour ce métier en dépit de la représentation féminine dont celui-ci jouit dans la société cana-dienne, comme d'ailleurs dans leurs sociétés d'origine ${ }^{12}$. Cependant, les entrevues réalisées postérieurement à notre enquête quantitative montrent que, loin d'être pour tous un choix délibéré et parfaitement assumé, ces individus subissent, en réalité, des contraintes dans l'accès au marché du travail dont la conséquence est souvent une forme de déclassement professionnel.

" Je dois vous dire sincèrement, quand je suis arrivé ici, j'ai travaillé dans des manufactures. J'ai travaillé comme trempeur de métal, j'ai travaillé comme apprenti machiniste, j'ai travaillé dans le tricot comme vérificateur de production. Ensuite, je suis retourné à l'école. C'està-dire, je suis allé en linguistique à I'Université et j'ai obtenu un diplôme. Ensuite... j'ai fait un certificat en andragogie. J'ai été dans l'enseignement, dans l'éducation des adultes. J'ai travaillé pendant sept ans. Bon... j'ai perdu mon travail par la suite, et il fallait 
quand-même que je trouve quelque chose d'autre à faire. J'ai fait des applications dans d'autres domaines, sauf que parfois, lorsque vous présentez votre curriculum vitae, on vous dit : " ici c'est pas votre place, c'est pas votre place, monsieur. On n'a pas de travail pour vous. » Donc... ma conjointe qui est infirmière m'avait conseillé d'aller suivre un cours de préposé et effectivement, c'est ce que j'ai fait. Et je me retrouve maintenant comme préposé aux bénéficiaires. »

«Dans mon pays, je travaillais dans l'éducation. J'enseignais les cours de physique à l'école secondaire. J'aimais ça. Mais quand on voyage... Je l'avais pas compris avant, je l'ai compris maintenant, mais là il est trop tard. » Pierre qui roule n'amasse pas mousse " on dit. Surtout quand on vient de ce genre de pays... pauvre donc on... On perd des... On recule un peu... En d'autres termes, il fallait tout recommencer le cycle. Si je voulais continuer dans l'enseignement il fallait que je recommence toutes les études et puis bon! J'avais besoin d'argent. C'est pour ça que je suis allé travailler à l'usine. Pour avoir de l'argent, donc. Et dire que j'étais professeur là-bas! De l'enseignement au travail en usine, c'est pas... On est pas dans le même domaine. C'était une façon de gagner un peu de sous... et puis finalement, j'ai pu trouver à moyen terme le métier d'auxiliaire familial... »

"Chez moi mon diplôme de travailleur social est reconnu. Mais en arrivant ici, je n'étais pas membre de la corporation des travailleurs sociaux du Québec alors j'ai laissé tomber le service social et j'ai passé trop de temps à travailler dans un autre domaine. Et quand j'ai perdu mon travail, au lieu d'aller à l'université pour avoir le bac en service social, parce que... parce j'ai des obligations, là, j'ai dit, je vais... choisir le métier, la profession d'auxiliaire. »

Et ce, même si de façon raisonnée, plusieurs mesurent encore cela comme une manière de privilège en regard du chômage ou du travail qu'ils ont exercé en usine.

"Ce qui me plaît le plus dans mon travail... c'est la liberté de pouvoir travailler et puis de ne pas être sur le bienêtre social. C'est ça! Bien que je ne gagne pas beaucoup d'argent. Mais quand-même, j'ai la liberté de travailler. Et puis, quand je touche... quand j'encaisse mon chèque, si petit qu'il soit, je suis satisfait. Parce que ça, je l'ai gagné à la sueur de mon front. Que vos enfants sachent que, quand vous sortez, vous allez travailler. Oui, et puis, quand votre femme vous demande quelque chose, vous êtes en mesure de le lui procurer, ça vous donne satisfaction. Mais si j'avais à choisir, c'est pas ça que je choisirais. »

\section{Genre, ethnicité et employeur}

La plupart des immigrants de notre échantillon travaillent pour le compte d'agences privées. Or, être salarié de l'un ou l'autre type d'entreprise se traduit par une inégalité dans les conditions de travail, à commencer par la précarité (emploi à la demande) et les échelles salariales - un auxiliaire d'agence gagne en moyenne 8 \$ de l'heure alors que ceux des Centres Locaux de Services Communautaires (CLSC) peuvent compter le double. En outre, les premiers doivent dégrever de leur propre budget les frais de transport ou encore l'achat de gants médicaux. Les auxiliaires de CLSC intègrent dans leur temps de travail, le temps de déplacement entre deux clients alors que leurs homologues d'agences ne sont rémunérés que sur le temps passé réellement au domicile du client. Ajoutons à cela l'absence de support professionnel. Ils ne reçoivent bien souvent qu'une information minimale, accèdent peu, voire pas du tout à la formation continue, et travaillent de façon isolée. Le contact avec leurs employeurs peut se résumer à un simple appel téléphonique pour annoncer un 
nouveau client ou l'arrêt d'un service.

II s'avère que $63 \%$ des effectifs des CLSC sont des individus nés au Canada de parents nés eux-mêmes au Canada ; les immigrés comptent pour $37 \%$. Dans les agences, le profil est quasiment inverse avec $57 \%$ d'immigrés. De plus, si on compare les lieux de naissance des immigrés, on observe là aussi une répartition non aléatoire. Les originaires de pays à majorité noire sont sur-représentés dans les agences alors que les individus en provenance de pays à majorité blanche accèdent à peu près à même hauteur que les Canadiens aux emplois dans les CLSC. Au total, les individus originaires de pays à majorité noire ou nés de parents euxmêmes originaires de pays à majorité noire comptent pour 18\% seulement des effectifs des CLSC alors qu'ils comptent pour $43 \%$ des effectifs des agences montréalaises. Autrement dit, un Canadien de « souche » a à peu près deux fois plus de chance de travailler pour un CLSC qu'une personne originaire d'un pays à majorité noire.

Le déplacement des immigrants pour venir pallier les besoins dans le secteur sanitaire n'est pas nouveau. Les politiques d'immigration sont, dans l'histoire du Canada, étroitement liées au marché interne du travail et son besoin de maind'œuvre (Miles, 1982 ; Calliste, 1993 ; Burawoy, 1976). Dans le secteur sanitaire, outre le recours à l'immigration spontanée, le Canada, comme beaucoup d'autres pays (ex : France, Angleterre, etc.), a puisé aux sources de ses ex-colonies pour répondre au besoin de main-d'œuvre qui allait croissant dans les années 50-60, période de grande expansion économique des pays industrialisés. Néanmoins, ces «déplacements» de population étaient savamment contrôlés de manière à assurer les besoins sans mettre en péril la situation des nationaux. Citons pour mémoire le déplacement des originaires des DOM-TOM en France métropolitaine pour venir occuper les emplois de catégories $C$ et $D$ de la fonction publique (Cognet, 1999) et l'entrée des infirmières des ex-colonies anglaises de la Caraïbe pour des emplois d'infirmières auxiliaires au Canada à la même période (Calliste, 1993). «L'invitation » des immigrants à venir prendre le «sale boulot» est d'abord révélateur de la poursuite d'un rapport colonial où, si les lieux peuvent avoir changé, le rapport de domination des nationaux sur les immigrés demeure, doublé par celui des Blancs sur les Noirs. Elle est aussi la mise en acte d'une représentation du travail en santé comme secteur d'activité divisé par la valeur sociale que l'on accorde à certains individus et aux patients qu'on leur confie. Certes, nous ne sommes plus dans les années 50-60 où les gouvernants pouvaient ouvertement se poser la question de l'opportunité de faire entrer sur le territoire canadien des infirmières noires pour s'occuper des populations «laborieuses» noires des grandes agglomérations: " In time it is difficult to resist the conclusion that there may be a limited field of service for qualified Negro nurses amongst the Negro minorities in the large centers $\gg 13$ ou encore pour combler les services psychiatriques ou les sanatoriums, secteurs peu prisés par les infirmières canadiennes blanches. Non, la rectitude politique en ce début de nouveau millénaire n'autoriserait plus une politique si ostensiblement colonialiste au parfum de discrimination et de racisme (Miles, 1982 ; Burawoy, 1976 ; Castles et Kosack, 1985). Non ! Et pourtant cela ne revient pas à dire que la gestion du travail en santé ne continue pas de s'appuyer sur des pratiques qui font la part belle aux hommes, un peu moins belle aux femmes mais bien plus mauvaise aux immigrés et surtout quand ceux-ci sont des femmes, noires de surcroît. Au Québec, comme dans d'autres provin- 
ces du Canada, les soins primaires et services à domicile sont en grande partie délégués aux femmes immigrantes noires (Neysmith, 1996 ; Cognet, 2000). Avec la restructuration du système de santé québécois, qui se traduit par la désinstitutionnalisation au profit du maintien à domicile et par un transfert accru des services au secteur privé, les agences de soins et services à domicile ne cessent de croître, si bien qu'il y a fort à parier que les accords de libre échange vont asseoir leur expansion (Poirier et al., 1998). Dans le courant de la mondialisation des échanges économiques, notons qu'à ce jour plusieurs agences montréalaises sont des filiales de multinationales qui voient dans le secteur de la santé la possibilité d'investissements très fructueux et en partie parce que les normes salariales qu'elles appliquent sont très loin d'offrir les mêmes conditions d'exercice du service public. Sur fond de réduction de coûts et de réorganisation du service public, la division du travail en santé ne fait qu'accentuer les lignes de partage entre les statuts, les compétences valorisées, le genre et l'ethnicité.

Dans notre comparaison des profils entre les auxiliaires familiales d'institutions publiques (CLSC) et les auxiliaires familiales d'agences privées, nous l'avons vu, les originaires de pays à majorité noire sont fortement surreprésentés dans les agences, mais cela est en particulier le cas des femmes de ce groupe d'origine : $63 \%$ des femmes noires exercent en agence alors qu'elles ne représentent au total que $28 \%$ de l'ensemble des femmes dans cette catégorie d'emploi. Les Noirs, hommes et femmes, sont plus nombreux que les Blancs dans la catégorie auxiliaire d'agence mais les hommes s'insèrent un peu mieux que les femmes en CLSC où les conditions de travail et de salaire sont plus confortables (42\% d'entre eux entrent en CLSC pour $38 \%$ de femmes). Aucune variable objective en termes de formation au métier, de niveau scolaire ou encore de connaissance des langues officielles (français et anglais) - facteurs listés comme pré-requis à l'embauche par les personnes en charge du recrutement dans les CLSC -, n'explique ces écarts ${ }^{14}$. Ces résultats associés à ceux de l'étude que nous avons menée en France ainsi qu'aux travaux dirigés par Neysmith et Aronson $(1996,1995)$ en Ontario nous amènent à considérer que si les femmes, en particulier les femmes immigrantes et a fortiori les immigrantes noires, sont conduites par la société dans ces catégories d'emploi, cela ne relève pas du hasard, mais procède de l'organisation de la société et de son système de santé. La formalisation de la délégation des actes, loin de résoudre cet aspect de la répartition des tâches, sert d'autel sur lequel se décident ces lignes de partage. Ceux qui participent au débat sont ceux qui, de par leur position dans le système, ont accès au pouvoir. La division sociale du travail en santé établie, il reste à observer comment cette division se manifeste dans son contenu.

Nos recherches nous conduisent à soutenir la thèse que la division du travail en santé repose en fait sur la délégation par un groupe professionnel à ceux qui lui sont subordonnés, d'activités ou de groupes particuliers de patients qu'il considère de moindre valeur, voire indigne de lui, pour reprendre les propos de Hughes (1955), du moins par la perception qu'il en a. De façon corrélée, il appert que les activités en question subissent un processus de dévaluation au cours du processus de délégation. On pourrait s'étonner que cette délégation soit derechef acceptée et assumée par les groupes subordonnés qui, quoi qu'en position de moindre pouvoir dans les organisations, n'en sont pas pour autant totalement démunis. Mais la délégation est en réalité facilitée par le fait que le groupe subor- 
donné voit là l'opportunité d'augmenter sa compétence et son champ d'action.

\section{La délégation d'actes dans le con- texte de l'évolution du système de santé québécois}

L'histoire professionnelle des infirmières est marquée par sa subordination au corps médical, d'où d'ailleurs est extraite la notion classifiante de profession « paramédicale », le préfixe assignant pour place à ces femmes d'être à côté des médecins. En se réservant le diagnostic et le traitement, les médecins déterminaient leur emprise hégémonique sur l'ensemble des activités de la santé puisqu'il s'agissait bien d'identifier les origines des maux et d'en soulager les malades. Le diagnostic et le choix du traitement leur appartenant, le reste n'était plus qu'une affaire d'exécution de leurs ordres. C'est assez récemment que cette délégation a été réglementée par des textes officiels. Au Québec, c'est à partir de 1973 que sept corporations professionnelles, dont les médecins et les infirmières, ont obligation de par leur loi constitutive d'arrêter par règlement la délégation d'actes sur lesquels leurs membres exercent un monopole. Le débat entre les différentes catégories concernées (médecins, infirmiers, infirmiers-auxiliaires) aboutira non sans heurts en 1980 à la promulgation des deux règlements de délégation des actes médicaux et infirmiers. Dans le même temps, cette ouverture de la délégation médicale va admettre ipso facto la délégation des infirmières à la catégorie qui leur est subordonnée. La tension entre les médecins et les infirmières, au cours des débats sur la formalisation de la délégation, n'est pas tant sur la part qu'ils vont reconnaître d'actes confiés aux infirmières - ils l'ont toujours fait, c'est la nature même de la relation du travail entre les deux groupes - que sur le travail que les infirmières entendent à leur tour déléguer aux autres catégories dans la mesure où il s'agit de prendre à leur compte une partie qui leur était autrefois déléguée, comme le nursing, pour en faire leur territoire sur lequel elles auront désormais autorité.

Bien que la délégation infirmière s'adresse spécifiquement aux infirmières auxiliaires et auxiliaires de puériculture, catégories visées par le règlement de 1980, le développement des soins à domicile sous l'égide des CLSC qui a largement ouvert l'institution aux auxiliaires familiales (certes moins rémunérées que les infirmières auxiliaires), a entraîné une dérive de la délégation. L'article 41 de la Loi sur les infirmières et les infirmiers exclut de l'interdiction de poser des actes compris dans le champs d'exercice exclusif de l'infirmière, les « personnes donnant des soins aux malades en qualité de domestique, de dame de compagnie, de bonne d'enfant ou d'aide domestique ». Pour pouvoir remplir leur mission, certains établissements publics et privés de la santé ont donc considéré les auxiliaires familiales comme des « aides domestiques » et ce même si la convention collective signée entre la direction des secteurs de la santé et services sociaux et les syndicats ne fait pas mention d'un rapport soignant entre auxiliaires familiales et clients. Les missions et tâches décrites se réduisent à une participation à l'entretien quotidien de la maison, la préparation et le service du repas, voire au besoin à des tâches familiales et des travaux domestiques légers et occasionnellement à une contribution au maintien du bien-être des bénéficiaires $^{15}$. Cette interprétation de la loi donne évidemment davantage préséance au lieu de l'exercice (le domicile) qu'au lien formel d'emploi (employés des CLSC et d'agences) ou encore à la formation ou aux connaissances pratiques et théoriques. 
Sans que soit formellement encadrée la délégation directe entre infirmières et auxiliaires familiales, on assiste néanmoins à un accroissement considérable des activités confiées à ces dernières (dont certaines n'ont reçu aucune formation puisqu'il n'y a encore à ce jour aucune exigence en la matière). En effet, on a pu observer que de nombreux actes réservés normalement aux infirmières et aux délégués sous leur contrôle aux infirmières auxiliaires, tels que l'administration de médicaments, la prise des constantes, les soins de stomies, les injections d'insuline, l'administration de lavements, les curages et touchers rectaux, l'aspiration de secrétions des voies respiratoires, etc., sont aujourd'hui pratiques courantes chez les auxiliaires familiales. Bien sûr, les auxiliaires familiales peuvent officiellement se référer au CLSC dont dépendent les malades, mais il faut compter que plus de la moitié du travail à domicile est sous-traitée par le privé. Les auxiliaires familiales travaillent de façon isolée sans référer à qui que ce soit leurs activités, sauf en de rares occasions.

Cet écart entre la loi et la pratique ainsi que l'argument pour le justifier (à savoir le déplacement des soins au domicile et l'assimilation des auxiliaires familiaux et sociaux au personnel domestique, Cf. article 41 de la Loi sur les infirmiers et les infirmières) n'est pas sans nous interpeller, eu égard au transfert accru d'une grande part des services et des soins aux secteurs privé et communautaire. En effet, il ne faut pas s'y tromper, ces négociations autour de la délégation ne sont compréhensibles que si nous les contextualisons dans la transformation des systèmes de santé. La partie ne se joue pas seulement entre les catégories professionnelles. Les enjeux en sont infiniment plus complexes. La dimension économique y joue un rôle prépondérant et c'est en définitive la valeur sociale que l'on accorde à tel ou tel état de santé - et conséquemment de façon inhérente à celui qui en est affecté - qui détermine les orientations et les choix ultimes. Le déplacement d'une grande partie des soins primaires - dont la plus grande part concerne les personnes âgées, les handicapés, les personnes en fin de vie ou atteintes de maladies chroniques évolutives et les malades mentaux ${ }^{16}$ des lieux d'hospitalisations à des structures alternatives et aux domiciles privés, entraîne avec lui un transfert d'un nombre grandissant d'actes à des catégories de salariés de moins en moins qualifiés dans des conditions de surveillance qui nous paraissent pour le moins discutables. Les soins que requièrent ces patients sont bien sûr moins de l'ordre des soins sophistiqués que du domaine de l'hygiène et de l'entretien de la vie courante. En dehors des aides à la toilette, au repas, à la prise de médicaments et au déplacement, leurs besoins se situent, pour la plupart, dans l'aide aux fonctions d'élimination (aspirations de sécrétions, lavements, changements de poches à urine, de poches à stomies), à prévenir ou limiter les complications ainsi que les risques inhérents à leur état (plaies de décubitus, pertes cognitives, etc.). Ce ne sont certes pas là les soins perçus par l'ensemble des professionnels de santé comme des plus agréables à exécuter, d'autant que les représentations communes les apparentent souvent au « sale » (Arborio, 1993).

La délégation est à analyser à la fois dans le contexte 1) de la rationalisation des services et donc des activités, 2) de la montée de la professionnalisation relative à l'augmentation des activités et de leur complexité, 3) de la tentative de résolution des tensions entre les catégories professionnelles dont les champs se recoupent sur de très larges zones. En 1970, la commission d'enquête sur la santé et le bien-être au Québec ${ }^{17}$ dénonçait les rapports de compétition 
interprofessionnelle, la course au statut et la hiérarchisation des activités (Dussault et Potvin, 1983). C'est bien dans un climat de compétition pourtant que se négocie encore aujourd'hui la formalisation des délégations, et en particulier celles qui concernent les auxiliaires familiales. Alors qu'un mémoire présenté par l'Association des Hôpitaux du Québec (AHQ), en décembre 200018 au groupe de travail ministériel sur les professions de la santé et des relations humaines, recommande l'élargissement de la délégation au profit des établissements et leurs directions au détriment de l'autorité tutélaire des groupes professionnels : "Remplacer en établissement, compte tenu de l'existence de mécanismes de contrôle de la qualité des soins et de l'encadrement professionnel qu'on y retrouve, le mécanisme de délégation des actes par un cadre général et des balises permettant aux établissements de décider euxmêmes ${ }^{19}$ eu égard à leur mission, des activités partageables à certaines conditions, applicables dans leur organisation. » À l'inverse, l'Ordre des Infirmières et des Infirmiers du Québec (OIIQ) ${ }^{20}$ souligne les dangers de la pratique en cours et appelle à inscrire la délégation aux auxiliaires familiales sous leurs autorité et compétences : "Les décideurs doivent toutefois se rendre à l'évidence que seule une évaluation professionnelle de l'ensemble de la situation de l'usager et non l'aspect gestuel d'un acte en soit doit conditionner la nature de l'activité de santé et orienter notamment l'ampleur de la contribution de divers intervenants. Faire abstraction de cette réalité ne peut que contribuer à augmenter les situations de prestations de soins sans encadrement et les risques de danger qu'elles peuvent engendrer pour la sécurité du public. Danger également que certaines instances continuent, à la lumière de conventions collectives ou de descriptions de tâches de certains travailleurs, à déterminer les actes infir- miers qui peuvent être posés par ces non-professionnels, et ainsi, en raison de leur méconnaissance du domaine clinique, à minimiser les nuances importantes qui doivent être faites entre les impératifs d'un milieu de soins et ceux d'un milieu de vie. " Par la voix syndicale $^{21}$, les infirmières auxiliaires entendent aussi faire valoir leur droit : « Les infirmières auxiliaires viennent tout juste d'obtenir, après un peu plus de six années de lutte acharnée, le droit de pratiquer les 16 actes délégués qui leur sont pourtant reconnus dans la loi. Bientôt de nouvelles négociations seront entreprises pour que leur soient délégués d'autres actes médicaux. Le 23 mai, elles manifesteront à Montréal afin d'amener les ministres Rochon et Marois à revoir leur décision de destiner les futures infirmières auxiliaires aux seuls soins de longue durée. "Si les auxiliaires familiales, qui ne bénéficient pas de la structure organisationnelle des professions, font difficilement entendre leur voix, on note, au cours des entretiens de notre enquête, des prises de position qui prêchent en faveur de l'extension de leurs territoires de compétences.

"Que ce soit sous la pression de la fédération des CLSC ou celle de l'Ordre infirmier, peu importe parce que les deux veulent que l'on soit mieux formé et que c'est la formation qui permettra de nous faire reconnaître comme professionnels. »

«De fil en aiguille, quand je travaillais avec les personnes âgées, souvent les infirmiers me disaient: » On aimerait te garder quelques heures de plus. "Ils se sont rendus compte que je pouvais prendre soin des gens, faire des pansements, etc., faire un suivi sur la santé, tout le tralala ; j'avais quand-même une base. »

« Vous allez voir un jour, nous serons en mesure d'aller plus loin : donner des piqûres ou... je sais pas, d'autres choses comme ça. Ce n'est pas encore arrivé, mais ça va venir vraiment. Parce 
qu'il faut des auxiliaires qui soient un peu plus reconnus dans le travail. »

Le débat n'est pas terminé et ne le sera sans doute pas avant longtemps puisqu'il traduit un rapport de force entre des fractions attachées à se partager un même objet. Dans le territoire délimité par I'OIIQ en regard de la formation infirmière, la part des activités que les médecins vont négocier va se référer au niveau de la complexité technique et du savoir spécialisé. C'est finalement selon un processus de catégorisation liée au savoir qu'est redéfinie la valeur des actes qu'ils se réservent ou qu'ils délèguent. Le processus est identique entre les infirmières et leurs subordonnées et c'est, du coup, avec une conscience plus aiguisée qu'aujourd'hui que les leaders infirmiers et leurs associations prennent position. L'acte de soin dépourvu de sa complexité et de l'ésotérisme du savoir qu'il supposait, n'est plus qu'une simple technique que l'on peut déléguer à des exécutants dont la qualification se borne à reproduire des gestes pensés et mis au point par d'autres. C'est sur cette toile de fond que se joue aujourd'hui le débat sur le nouveau projet des actes infirmiers au Québec 22 et en particulier sur des questions comme la possibilité de con-fier, par exemple, l'administration de gavage par voie nasogastrique ou par gastrostomie, d'effectuer des stimulations anales ou encore de réaliser un cathétérisme vésical intermittent - actes réservés depuis 1981 aux infirmières ${ }^{23}$ - et délégués aux personnes autres que des infirmiers et infirmières.

\section{CONCLUSION}

Au total, il appert que la délégation, au-delà de ses objectifs explicites, prend place au cœur d'un triple rapport de domination par le genre, le statut migratoire et l'appartenance ethnique.
Non seulement elle ne peut annihiler ce rapport de domination mais on constate même qu'elle contribue à l'entretenir. Ce sont des actes dévalués, moins prestigieux, touchant la matière moins noble qui sont délégués, plus ou moins explicitement d'ailleurs, aux catégories professionnelles subalternes des organisations de santé. Ce sont aussi des groupes de patients (personnes âgées, handicapés, personnes en fin de vie, atteintes de maladie chronique et malades mentaux) qui leur sont confiés, l'état de santé de ces derniers ayant peu de chances de s'améliorer. Ils ne posent pas ou plus de défi majeur à la médecine ; l'objectif maximal pour la plupart d'entre eux relève de l'entretien et du palliatif. La division du travail en santé est ainsi affaire d'enjeux à la fois professionnels, organisationnels et économiques. D'abord professionnels, puisqu'ils exercent une action déterminante sur la position des groupes. II est clair qu'au Québec les infirmières ont profité des débats sur les actes professionnels pour renforcer leur identité et marquer leur émancipation du corps médical en délimitant un territoire propre, celui du nursing puis, plus tard, celui du Care par opposition à celui de Cure, réservé aux médecins. Pour les catégories subordonnées telles que les infirmières auxiliaires ou les auxiliaires puéricultrices, l'enjeu réside dans la tentative de gagner leur indépendance visà-vis des infirmières. En cela, elles ont à différentes reprises bénéficié du soutien des médecins - qui, de leur côté, ont tout intérêt à limiter l'expansion des infirmières qui grignotent leur territoire - et des gouvernants qui, en leur accordant plus de marge de manœuvre contre l'intérêt des infirmières, font une économie directe. Enfin, dans les catégories encore moins bien représentées dans le système, telles que les auxiliaires familiales où les femmes immigrantes noires sont les plus nombreuses, si là aussi l'enjeu réside dans l'obtention d'une meilleure reconnaissance de compé- 
tence par l'appropriation d'actes, elles sont néanmoins dans des positions d'autant plus difficiles pour négocier qu'elles sont maintenues à distance des processus et des lieux de la décision. Mais les enjeux dépassent l'intérêt des catégories professionnelles pour mettre en scène les organisations de santé avec pour arrière fond les intérêts écono-miques de la société. Pour les nouvelles entreprises publiques et privées qui assurent le « virage ambulatoire », les enjeux de la délégation sont le nerf de la guerre. Pour maintenir leurs coûts au plus bas, il faut qu'elles obtiennent plus de marge d'action pour leurs employés et qu'elles limitent le recours à des professionnels plus qualifiés. La main-d'œuvre immigrée, qui s'offre spontanément sur leur marché est, telle la manne céleste, des plus opportunes pour atteindre leurs objectifs. Dans le cas des soins primaires, l'émergence des CLSC redistribue les cartes entre les organisations de santé sur ce même modèle. L'option qui a été prise de ne pas recruter des infirmières auxi-liaires au profit d'une catégorie moins qualifiée en termes de formation (les auxiliaires familiales) mais aussi plus faiblement rémunérée (a fortiori dans le recours à la sous-traitance avec des agences privées), conduit aujourd'hui à une pratique de délégation envers ces dernières que l'Ordre des infirmiers tente à ce jour d'endiguer. II devra compter avec le poids qu'ont pris aujourd'hui ces entreprises publiques et privées (dont un certain nombre sont des filiales de holdings internationaux) et leurs directions qui, tout en maintenant leur pression pour occuper le terrain des soins primaires, continueront de devoir répondre à des objectifs de rationalités des coûts.

\section{Notes}

1 Nous nous référons à une série de travaux réalisés en France (M. Cognet, 1999) et au Québec a) - D. Meintel, M. Cognet, L. Raigneau, R. Antonius, J. Renaud, S. Fortin et R. Sévigny (coll.), « Les auxiliaires familiaux en CLSC : ethnicité, formation et insertion institutionnelle » étude financée par le CQRS ; b) - M. Cognet, J. Rhéaume, D. Meintel, J. Renaud, L. Raigneau, « Infirmières et travailleurs sociaux. Entre modèles professionnels et modèles culturels » étude financée par l'Institut de Recherche en Santé du Canada (IRSC).

2 Dans le cadre de cette présentation, nous n'entrerons pas dans le débat concernant ces deux notions de «professions » et « métiers », mais outre une série de travaux qui font le point sur la question tels que la synthèse de Claude Dubar et Pierre Tripier (1998), signalons l'étude très stimulante de Michèle Descolonges (1996) qui au travers de l'évolution de l'une et l'autre des notions, réintroduit de façon centrale l'idée de maîtrise de l'art.

3 Compte tenu justement du poids des femmes dans les catégories paramédicales nous adopterons le féminin tout au long de ce texte.

4 Sources Statistique Canada http://www.statcan.ca/start_f.html

5 Sources OIIQ www.oiiq.org

6 Comité directeur de l'étude sectorielle sur le travail social, Développement des ressources humaines Canada, Le travail social au Canada: une profession essentielle, Rapport final, Volume 1, janvier 2001.

7 Les résultats présentés sont extraits d'une étude concernant plus spécifiquement la situation des auxiliaires familiaux qui exercent aujourd'hui à Montréal. Nous ferons référence aux analyses effectuées : 1) à partir de données recueillies par questionnaire sur un échantillon de 181 individus travaillant auprès de la clientèle des services de Maintien à domicile des Centres Locaux de Services Communautaires (CLSC) mais qui peuvent être autant leurs propres employés que ceux d'agences privées avec lesquelles les CLSC engagent une sous-traitance. Notre échantillon a été recruté auprès de trois CLSC et de trois agences privées de Montréal ; 2) à partir d'une enquête par entrevues semi-directives réalisées post-questionnaire auprès de 40 auxiliaires recrutés selon des critères définis à partir de cinq profils de trajectoire analysés avec le questionnaire considérant le genre, l'ethnicité, l'employeur, les trajectoires professionnelles dans le métier et antérieures). 
8 Au Québec, les immigrants représentent 9,4\% de la population totale de la province mais pour la seule région administrative de Montréal, ils comptent pour 26,5\% (Sources MRCI Statistiques, données issues du recensement de 1996).

${ }^{9}$ Au total, sur un échantillon visé de 280 individus, 181 ont rendu un questionnaire valide pour traitement statistique (65\%) Toutefois ce taux de réponses est inégal entre les deux types d'employeur pour lesquels il était souhaité une répartition à 50\%. Dans les CLSC, le taux est de $77 \%$ alors que dans les agences il n'est que de $52 \%$. Ce moindre retour est imputable aux difficultés à rejoindre les employés, d'une part parce qu'une large partie de cette population ne se déplace pas sur le lieu de l'agence (la passation devait se dérouler face à face enquêteur/enquêté) et d'autre part elle est pour l'ensemble très mobile, ce qui n'a pas permis d'atteindre les objectifs initiaux, en dépit des informations du passage des enquêteurs et des jours d'enquête.

10 Il n'existe pas actuellement de données chiffrées sur la population des auxiliaires familiales à Montréal et ce, en grande partie, parce qu'il s'agit d'une population très mobile et dont le temps de travail hebdomadaire peut varier de 1 à 50 heures. Néanmoins, nous prenons pour base de sondage la totalité des membres enregistrés à l'association des auxiliaires familiales du Québec qui compte 740 individus.

11 Pour l'ensemble des individus qui ont déclaré être nés au Canada, la majorité est née au Québec (92\%).

12 Plusieurs de ces hommes préciseront au cours des entrevues que, pas plus dans les pays d'origine qu'ici, ce type de travail n'est apparenté au masculin, il peut même être perçu comme dévalorisant pour un homme de s'y adonner.

13 Extrait d'une lettre du responsable du Département canadien de l'immigration à la Présidente de l'Association des infirmières canadiennes (CNA) en 1950, cité dans Calliste (1993).

14 Les calculs sont effectués sur notre échantillon avec le test de Pearson Chi-Square sur lequel, en cas de seuil significatif, s'applique le calcul de mesure directionnelle au moyen du Lambda et du Goodman and Kruskal tau. En l'occurrence le Khi2 n'atteste pas de relation entre la couleur de la peau et ces autres variables.

15 Cf. Convention collective, 2000-2002, CPNSSS-CSN, nomenclature des titres d'emplois, des libellés et des échelles de salaires. En fait, le syndicat (CSN) s'oppose à l'interprétation de l'article 41 faite par les établissements. Pour eux, indépendamment du lieu d'exécution des tâches, les auxiliaires sont des employés des CLSC (Cf. Fédération des affaires sociales (CSN) en collaboration avec le Comité CLSC/Centres de santé et le service de l'information de la CSN, Aide-mémoire des auxiliaires familiales et sociales, octobre 1996, 11 p.).

16 Le Québec, pour sa part, a procédé en 1989 à une désinstitutionnalisation massive des patients psychiatrisés.

17 Commission Castonguay-Nepveu, vol. IV, tome I, 1ère partie.

18 Association des Hôpitaux du Québec (A.H.Q.), décembre 2000, Mémoire sur la modernisation de l'organisation professionnelle de la santé et des relations humaines, présenté au groupe de travail ministériel sur les professions de la santé et des relations humaines.

\section{Souligné par nous.}

20 OIIQ, mai 2001, La vision contemporaine de l'exercice infirmier au Québec, mémoire présenté au groupe de travail ministériel sur les professions de la santé et des relations humaines dans le cadre de la mise à jour du système professionnel.

21 Nouvelles CSN, numéro 442, 8 mai 98, http://www.csn.qc.ca

22 OIIQ, mai 2001, op. cit.

23 Règlement sur les actes visés à l'article 31 de la loi médicale qui peuvent être posés par des classes de personnes autres que des médecins, décisions 18-09-1981 (L.R.Q., c. M-9, a. 19, 1er al. Par. b), (L.R.Q., c. C-26.). 


\section{Bibliographie}

ARBORIO, A-M.,1993. "Quand le "sale boulot " fait le métier : les aides soignantes dans le monde professionnalisé de l'hôpital », Sciences sociales et santé, 13, 3, pp. 11-25.

ARONSON, J. et NEYSMITH, S., 1996. « The Work of Visiting Homemakers in Context of Cutting in Long Term Care ", Canadian Journal of Public Health, 87, 6, pp. 422-425.

BLONDEAU, D., 1993. « Les valeurs de la profession infirmière d'hier à aujourd'hui », in Goulet, O. (dir.), La profession infirmière : valeurs enjeux, perspectives, Gaétan Morin, Québec, pp. 7-22.

BUCHER, R., STRAUSS, A., 1961. « Professions in Process », The American Journal of Sociology, 66, 4, January, pp. 325-334.

BURAWOY, M., 1976. « The Functions and the Reproduction of Migrant Labor ", American Journal of Sociology, 81, 5, pp. 1050-1087.

CALLISTE, A., 1993. "Women of « Exceptional Merit »: Immigration of Carribean Nurses of Canada », Canadian Journal of Women and Law, 6, pp. 85-102.

CASTLES, S., KOSACK, G., 1985. Immigrant Workers and Class Structure in Western Europe, Oxford University Press, Oxford, (première ed, 1973), $534 \mathrm{p}$.

COGNET, M., 2000. « Les auxiliaires familiaux et sociaux : domestiques ou soignants ? », Communication présentée au XVle congrès de I'AISLF, Université Laval, Québec.
COGNET, M., 1999. « Migrations, groupes d'origines et trajectoires : vers une ethnicisation des rapports socioprofessionnels ? " Thèse de Doctorat de Sociologie, Éditions des Presses Universitaires du Septentrion, Villeneuve d'Ascq, $306 p$.

COHEN, Y., 1995. « La modernisation des soins infirmiers dans la province de Québec (1880-1930). Un enjeu de négociation entre professionnels », Sciences Sociales et Santé, 13, 3, pp. 11-33.

CROZIER, M. ET FRIEDBERG, E., 1977. L'acteur et le système, Paris, Seuil, Points, $500 \mathrm{p}$.

DESCOLONGES, M., 1996. Qu'est-ce qu'un métier ?, PUF, Paris, 264 p.

DESROSIERS, G., 2001. « Les professions de santé sous examen » in $\mathrm{H}$. Morais et M. Venne, (dir.) Santé : pour une thérapie de choc, Les Presses de I'Université de Laval, Québec, pp. $87-$ 92.

DUBAR, C., 2000. La socialisation. Construction des identités sociales et professionnelles, Armand, Colin, Collection $U$, Paris, [première ed, 1991]. 276 p.

DUBAR, C., TRIPIER, P., 1998. La sociologie des professions, Armand, Colin, Collection U, Paris, 256 p.

DUSSAULT, G., POTVIN, L., 1983. L'État et la division du travail sanitaire. La politique de délégation des actes médicaux, infirmiers et dentaires au Québec, Département des relations industrielles, Université Laval, $152 \mathrm{p}$.

FREIDSON E., 1984. La profession 
médicale, Paris, Payot,. [1970, Profession of Medicine, New York, Harper and Row, trad], 369 p.

GLAZER, B., STRAUSS, A., 1964. «The Social Loss of Dying Patients », American Journal of Nursing, 64, 6, June, pp. 119-121.

HERZLICH, C., BUNGENER, M., PAICHELER, G., ZUBER, M-C., 1993. Cinquante ans d'exercice de la médecine en France. Carrières et pratiques des médecins français (1930-1980), CERMES, Paris, INSERM-Doin.

HUGHES, E.C., 1955. «The Making of a Physician», Human Organization, III, 1955, pp. 21-25 repris dans Men and their Work, Glencoe, The Free Press, [2 éd. 1967], ch. 9, pp. 116-131.

HUGHES, E.C., 1958. Men and their Work, Glencoe, The Free Press, $2^{\circ}$ éd., 1967.

MILES, R., 1982. Racism and Migrant Labour, Routledge and Kegan Paul, London, 202 p.

MONTGOMERY, C., COGNET, M., TREMBLAY, L., XENOCOSTAS, S., 2001. "Les faces cachées du système de santé » in $\mathrm{H}$. Morais et $\mathrm{M}$. Venne, (dir.) Santé : pour une thérapie de choc, Les Presses de I'Université de Laval, Québec, pp. 4752.

NEYSMITH, S, 1996. " Les soins à domicile et le travail des femmes : la force de l'habitude », Lien Social et Politiques - RIAC, 36, pp. 72-80.

NEYSMITH, S. ET ARONSON, J., 1995. Working Conditions in Home Care : Negotiating Race and Class
Boundaries in Gendered Work, Paper presented at the Meetings of the Canadian Association on Gerontology. Vancouver, Canada, Octobre.

PAICHELER, G., 1995. " Les professions de soins : territoires et empiétements », Sciences Sociales et Santé, 13, 3, Septembre, pp. 5-10.

PAICHELER, G., 2000. « Femmes et hommes dans le champs de la santé », Recherche-Santé-Social (ENSP), pp. 179-196.

POIRIER, M., PATENAUDE, F., PETIT M., LAMBERT, G., 1998. À qui profite le démantèlement de l'État ?, Chaire d'études socio-économiques de I'UQAM, Montréal, 306 p.

SAILLANT, F., (1994). " La part des femmes dans les soins de santé », Revue Internationale d'Action Communautaire (RIAC), 28, 68, 95106.

SOLOMON, D. N., 1961. « Ethnic and Class Difference among Hospitals as Contingencies in Medical Careers », American Journal of Sociology, 66, 5, pp. 412-437.

STRAUSS, A., 1963. « Hospital and its Negociated Order », in Freidson E, The Hospital in Modern Society, New York, The Free Press, pp. 147-168.

TRABACCHI, G., 2000. «Réactualisation du décret de compétence relatif à la profession », Soins, 642, JanvierFévrier, pp. 7-9. 